\title{
A new algorithm for detecting cloud height using OMPS/LP measurements
}

\author{
Zhong Chen ${ }^{1}$, Matthew DeLand ${ }^{1}$, and Pawan K. Bhartia ${ }^{2}$ \\ ${ }^{1}$ Science Systems and Applications, Inc., 10210 Greenbelt Road, Suite 600, Lanham, Maryland 20706, USA \\ ${ }^{2}$ NASA Goddard Space Flight Center, 8800 Greenbelt Road, Greenbelt, Maryland 20771, USA \\ Correspondence to: Zhong Chen (zhong.chen@ssaihq.com)
}

Received: 30 July 2015 - Published in Atmos. Meas. Tech. Discuss.: 2 October 2015

Revised: 9 March 2016 - Accepted: 11 March 2016 - Published: 23 March 2016

\begin{abstract}
The Ozone Mapping and Profiler Suite Limb Profiler (OMPS/LP) ozone product requires the determination of cloud height for each event to establish the lower boundary of the profile for the retrieval algorithm. We have created a revised cloud detection algorithm for LP measurements that uses the spectral dependence of the vertical gradient in radiance between two wavelengths in the visible and near-IR spectral regions. This approach provides better discrimination between clouds and aerosols than results obtained using a single wavelength. Observed LP cloud height values show good agreement with coincident Cloud-Aerosol Lidar and Infrared Pathfinder Satellite Observation (CALIPSO) measurements.
\end{abstract}

\section{Introduction}

The Ozone Mapping and Profiler Suite Limb Profiler (OMPS/LP) is one of three OMPS instruments on board the Suomi National Polar-orbiting Partnership (S-NPP) satellite (Flynn et al., 2007). S-NPP was launched in October 2011, into a sun-synchronous polar orbit. The local time of the ascending node of the S-NPP orbit is 13:30. The LP instrument collects limb-scattered radiance data and solar irradiance data on a 2-D charge coupled device (CCD) array over a wide spectral range $(290-1000 \mathrm{~nm})$ and a wide vertical range $(0-80 \mathrm{~km})$ through three parallel vertical slits. Each slit provides a $1.85^{\circ}$ vertical field of view (FOV) corresponding to a $112 \mathrm{~km}$ vertical extent at the tangent point. The FOV of each slit is separated horizontally by $250 \mathrm{~km}$ in the cross-track direction. The OMPS/LP produces three ozone profiles every $19 \mathrm{~s}$ along the orbit track, which cor- responds to a sampling distance of about $150 \mathrm{~km}$ (approximately $1^{\circ}$ latitude). OMPS/LP has been operating continuously since April 2012, collecting approximately 160-180 measurements (events) per orbit for each of the three slits and each of the 14-15 orbits per day. Jaross et al. (2014) provide more details about the OMPS/LP instrument design and capabilities.

Retrieval of ozone profiles from limb-scattering measurements becomes extremely difficult in the presence of tropospheric clouds, because these clouds shield the signal from the lower atmosphere and also reflect a part of the incoming radiation back to space. Due to the potential bias in the retrieved profiles from clouds, the OMPS/LP retrievals are based on a cloud-free assumption. Thus, the current ozone retrieval algorithm applied to LP measurements is designed to identify cloud height (if present) for each event and to terminate the retrieval $1 \mathrm{~km}$ above this height.

Several techniques to retrieve cloud information from remote-sensing measurements have been developed. Most of them use changes in the oxygen A-band where the absorption of oxygen is sensitive to cloud-top height for retrieving cloud information (Kuze and Chance, 1994; Koelemeijer et al., 2001; Rozanov and Kokhanovsky, 2004; Bourassa et al., 2005; Eichmann et al., 2016; Kokhanovsky et al., 2005; von Savigny et al., 2005; Loyola et al., 2007, 2010; van Diedenhoven et al., 2007; Schuessler et al., 2014). Many of these algorithms need a forward model with necessary assumptions to solve the radiative transfer equation in a multilayer, multiple-scattering, and absorbing atmosphere. In view of the OMPS/LP sensor relatively coarse spectral resolution (10 $\mathrm{nm}$ in visible region), Rault and Loughman (2013) determine cloud height based on the identification of a sharp 
change in the vertical gradient of visible or near-infrared radiances. Clouds appear as either faint or sharp discontinuities in the reflected sunlight radiance vertical profiles. However, aerosol layers can also cause relatively abrupt changes in the radiance profile at visible and IR wavelengths, so this approach cannot always differentiate between tropospheric cloud and aerosols.

This paper describes a revised approach to cloud-top height detection using OMPS/LP measurements, based on the spectral dependence of the vertical gradient in radiance between two wavelengths. The approach is simple to implement. It is capable of distinguishing between aerosols and cirrus clouds in many cases. We show that the performance of this approach is consistent with Cloud-Aerosol Lidar and Infrared Pathfinder Satellite Observation (CALIPSO) results for quasi-coincident orbits in individual cases, as well as for a larger statistical comparison.

\section{Algorithm design}

The new gradient-based LP cloud detection algorithm assumes that clouds produce a larger gradient in radiances than aerosols. Because of the different size distributions between aerosol particles and cloud hydrometeors, their scattering of incoming solar radiation shows a different behavior. At UV and shorter visible wavelengths, Rayleigh scattering reduces the contrast between cloudy and clear pixels. This contrast increases with longer visible and near-IR wavelengths. Since aerosol particles are smaller, their increase in brightness is less pronounced for the same change in wavelength, so the increase in contrast for aerosols is not as large as for clouds.

We define the vertical gradient of observed radiances as the rate of change in radiances with tangent height:

$G(\lambda, z)=\partial \ln I(\lambda, z) / \partial z$,

where $I(\lambda, z)$ is the limb radiance as a function of wavelength $\lambda$ and tangent height $z$. The variation of the radiance gradient (i.e., the height derivative) with wavelength between 500 and $900 \mathrm{~nm}$ for various targets is shown in Fig. 1. Clearsky scenes show Rayleigh scattering with almost no wavelength dependence, as expected. Note that the tropospheric cloud at $14.5 \mathrm{~km}$ shows a steeper wavelength dependence than the aerosol layer at $25.5 \mathrm{~km}$. At visible and near-IR wavelengths longer than $\lambda_{\mathrm{s}}=674 \mathrm{~nm}$, where the absorption of light by ozone can be neglected, the dependence of the radiance gradient on wavelength can be parameterized using a linear relationship.

$G(\lambda, z) \approx \alpha(z)\left(\lambda-\lambda_{\mathrm{s}}\right)+k(z) ; \lambda \geq \lambda_{\mathrm{s}}$,

where $\alpha$ and $k$ - the slope and intercept, respectively - are a function of $z$ and independent of wavelength $\lambda$. Thus, the absolute value of $\alpha$ is the measure of the strength of the spectral variation in radiance gradient. The slope $\alpha$ in Eq. (2) can be

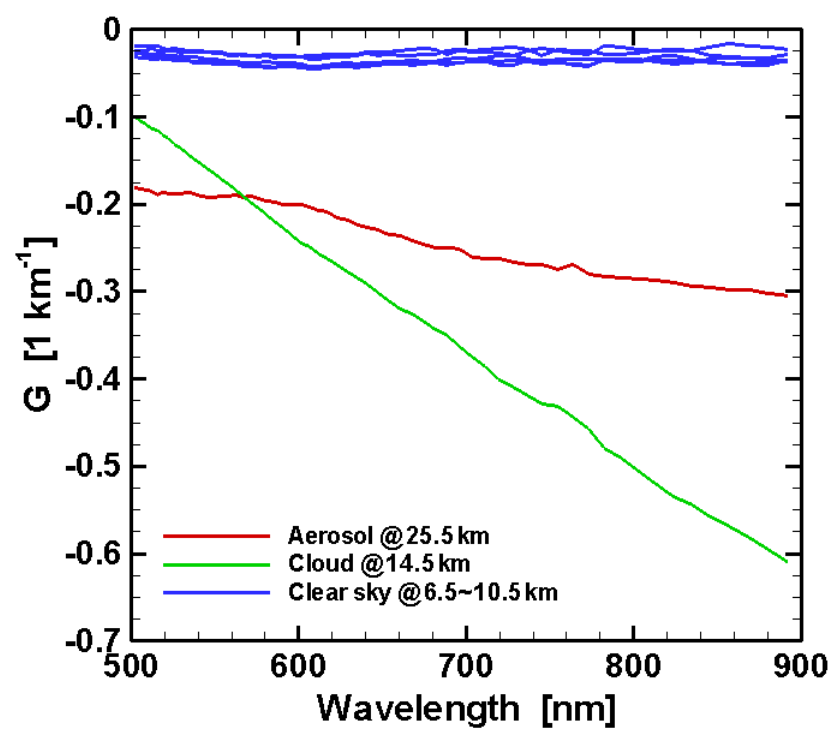

Figure 1. Variations in the radiance gradient $G(\lambda, z)$ from OMPS/LP data at $5^{\circ} \mathrm{S}$ during orbit 16754 on 21 January 2015. Blue: clear sky; green: cloud; red: aerosol.

determined by choosing a longer wavelength $\left(\lambda_{1}\right)$ :

$\alpha(z)=\left[G\left(\lambda_{\mathrm{s}}, z\right)-G\left(\lambda_{1}, z\right)\right] /\left(\lambda_{\mathrm{s}}-\lambda_{1}\right)$.

We choose the two wavelengths $\lambda_{\mathrm{s}}$ and $\lambda_{1}$ within the LP measurement range to maximize the cloud signature. As the shorter wavelength $\lambda_{\mathrm{s}}, 674 \mathrm{~nm}$ is chosen to avoid Chappuis band ozone absorption. Data rate limitations on the S-NPP spacecraft mean that not all possible wavelengths and altitudes measured by LP can be downloaded during regular operations. Although LP measurements extend to $\sim 1015 \mathrm{~nm}$, changes in spectral coverage during the S-NPP mission mean that $868 \mathrm{~nm}$ represents the longest wavelength $\lambda_{1}$ which is used in Eq. (3) available with full temporal coverage.

Calculating the slope values for the cases shown in Fig. 1, we find that $\alpha(6.5 \sim 10.5 \mathrm{~km}) \approx 0$ is consistent with the spectrally independent gradient expected for clear sky, $\alpha(25.5 \mathrm{~km})=-0.00027$ represents the weaker spectral dependence of radiance gradient for an aerosol, and $\alpha(14.5 \mathrm{~km})=-0.0013$ corresponds to the strongest spectral dependence of radiance gradient for a cloud. We note that, since the slope values are typically negative, we can rewrite Eq. (3) to define the gradient difference $\ln \mathrm{R}(z)$ :

$\ln \mathrm{R}(z)=\left[G\left(\lambda_{\mathrm{s}}, z\right)-G\left(\lambda_{1}, z\right)\right]=\alpha(z)\left(\lambda_{\mathrm{s}}-\lambda_{1}\right)$.

Identifying the largest values of the gradient difference $\ln \mathrm{R}$ in a measured profile should therefore provide a sensitive indicator for the presence of clouds. 

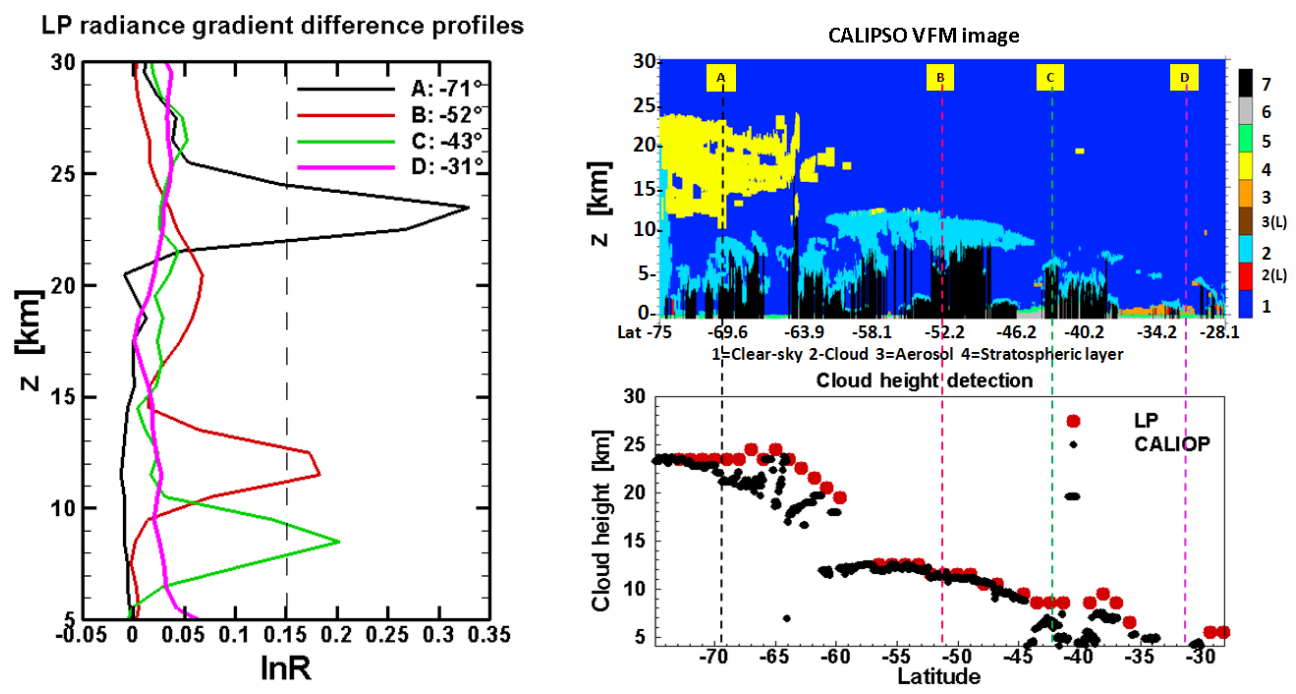

Figure 2. Radiance gradient and cloud detection results for four Southern Hemisphere events from a single orbit on 16 August 2012, using OMPS/LP measurements and CALIPSO vertical feature mask (VFM) daytime data. Left panel: vertical profiles of the LP radiance gradient $\ln \mathrm{R}$ for each event. The dashed black line represents the cloud detection threshold, which identifies clouds in events A, B, and C. Top right panel: CALIPSO VFM image for the same orbit. Yellow features indicate polar stratospheric clouds (PSCs), and light blue patches represent clouds. Bottom right panel: cloud height values detected by the LP algorithm (red dots) and from CALIPSO VFM data (black dots) in the same orbit. The four colored dotted lines in the right panel indicate the four events labeled as A, B, C, and D.

\section{Results}

\subsection{Threshold determination}

The method described in Sect. 2 has been used to determine cloud-top height from OMPS/LP measurements. We assign a positive cloud detection if the value of $\ln R$ in Eq. (2) meets a threshold value $F$ at some altitude in the radiance gradient profile. To determine the cloud detection threshold, we use CALIPSO $532 \mathrm{~nm}$ backscattering daytime data (Winker et al., 2003) and the corresponding CALIPSO vertical feature mask (VFM) version 3 data product (Vaughan et al., 2004; Kacenelenbogen et al., 2011) on selected days where the satellite tracks of Suomi NPP and CALIPSO most closely overlap. Figure 2 provides an example of the determination of $F$ during S-NPP orbit 4163 on 16 August 2012 for three events with clouds as well as one event without a cloud. These events show distinctly different signatures in their $\ln \mathrm{R}$ profiles. The sharpest vertical gradient, with a maximum value of $\ln \mathrm{R}=0.33$ at $23.5 \mathrm{~km}$, is observed for a polar stratospheric cloud (PSC). For clouds at lower altitudes, the maximum values of $\ln \mathrm{R}$ fall between 0.18 and 0.20 . However, for the clear-sky event, the maximum value of $\ln R$ is very small (less than 0.05). Further comparisons with CALIPSO observations indicate that $F=0.15$ is a reasonable threshold for positive cloud detection in LP data.

\subsection{Influence of aerosols}

Confirming the presence of a cloud at any altitude requires an ability to discriminate between cloud and aerosol signals.
We define a quantity called aerosol scattering index (ASI) at $674 \mathrm{~nm}$ for detecting aerosols in LP measurements:

$\mathrm{ASI}=\left(I_{\mathrm{m}}-I_{\mathrm{c} 0}\right) / I_{\mathrm{c} 0}$,

where $I_{\mathrm{m}}$ is the measured radiance and $I_{\mathrm{c} 0}$ is the calculated radiance using a forward model (Herman et al., 1995) for a Rayleigh atmosphere. Both $I_{\mathrm{m}}$ and $I_{\mathrm{c} 0}$ are normalized at $45 \mathrm{~km}$, assuming that there is no aerosol at that altitude. Figure $3 \mathrm{a}$ shows aerosols at $20-22 \mathrm{~km}$ at tropical latitudes, identified using ASI values for a single orbit on 19 June 2014. Although ASI is sensitive to stratospheric aerosols, ASI values also increase in the presence of clouds, so that this quantity alone does not distinguish between aerosols and clouds. Figure $3 \mathrm{~b}$ shows clouds at $10-15 \mathrm{~km}$ identified by CALIPSO data for the same event. In the CALIPSO image, the red-gray-white colored features indicate clouds between 10 and $15 \mathrm{~km}$ detected by lidar data, and the red dots represent LP cloud height values detected by our new algorithm for the same orbit. Note that the LP cloud locations are consistently at the top of the CALIPSO cloud regions. Figure $3 \mathrm{c}$ illustrates the LP radiance gradient profiles for a single event at $3^{\circ} \mathrm{S}$, identified by the dashed line in Fig. 3a and b. Note that $G(868 \mathrm{~nm})$ shows peaks of comparable magnitude at $12.5 \mathrm{~km}$ (tropospheric cloud) and $21.5 \mathrm{~km}$ (aerosol), whereas $G(674 \mathrm{~nm})$ has a similar magnitude peak at $21.5 \mathrm{~km}$ but a smaller peak at $12.5 \mathrm{~km}$. Thus, the gradient difference $\ln \mathrm{R}$ clearly identifies the maximum cloud altitude using the threshold specified in Sect. 3.1 and does not select the aerosol layer. 
(a) ASI (674)

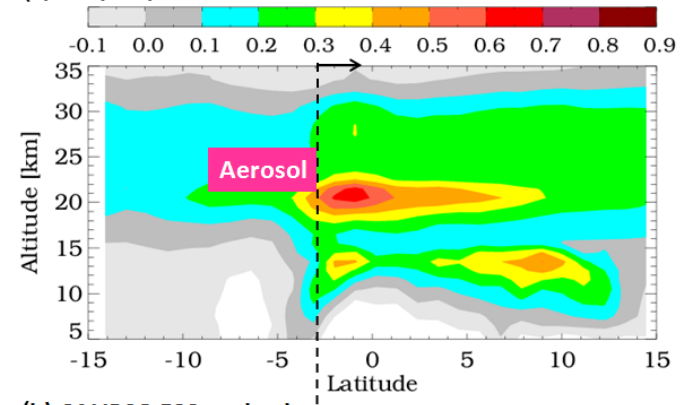

(b) CALIPSO 532nm backsóatter

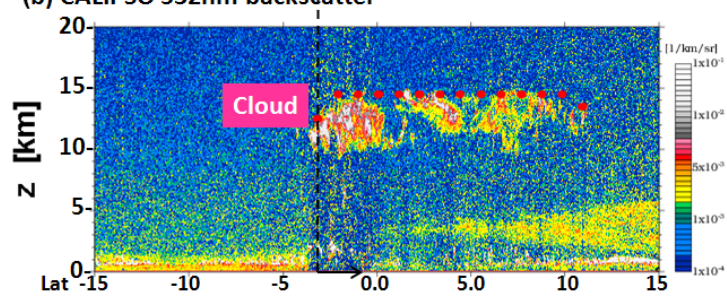

(c) Radiance gradient at 3.1 south

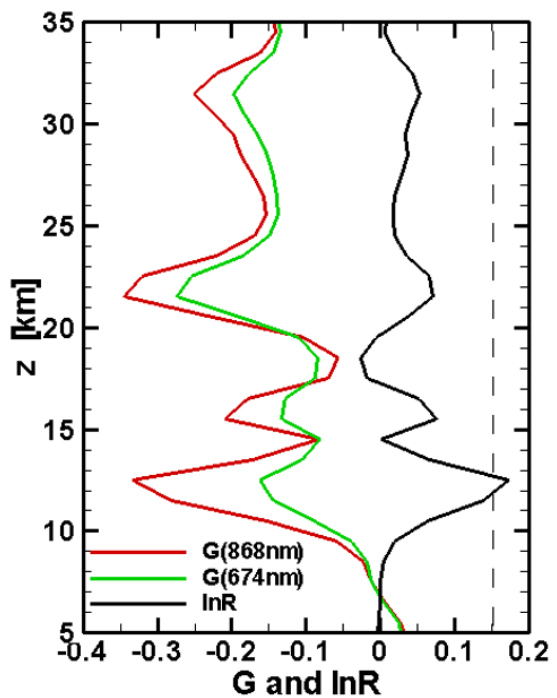

Figure 3. Example of discrimination between clouds and aerosols, using OMPS/LP observations taken on 19 June 2014. (a) Aerosol layer at 20-22 km in tropics identified using OMPS/LP aerosol scattering index (ASI). (b) Tropospheric clouds at 10-15 km identified in CALIPSO data for the same orbit. The red dots represent LP cloud-top height values derived from the radiance gradient algorithm. (c) LP radiance gradient profiles (red: $868 \mathrm{~nm}$; green: $674 \mathrm{~nm}$ ) for a single event at $3.1^{\circ} \mathrm{S}$, identified by the dashed line in panels (a) and (b). The difference between profiles (ln R) is shown as the black line.

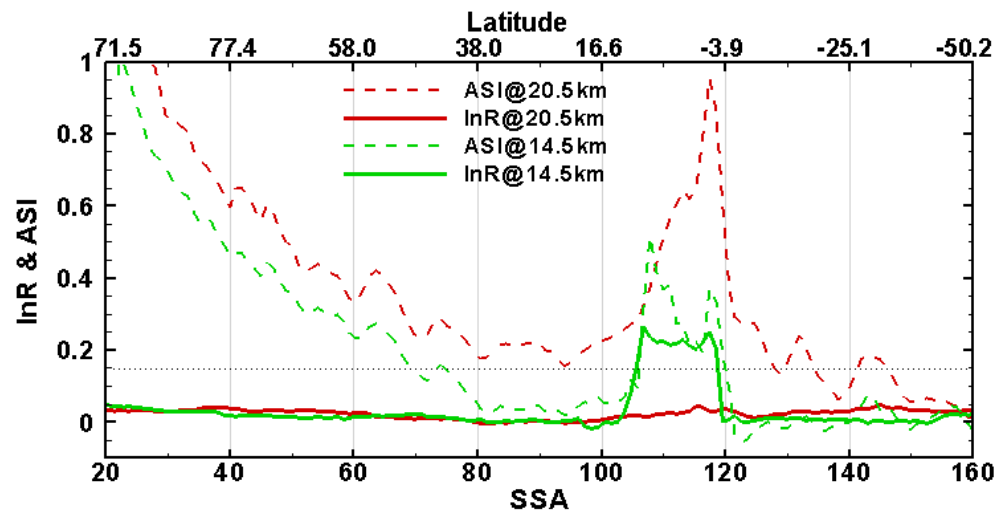

Figure 4. $\ln \mathrm{R}$ (solid line) and ASI (dashed line) plotted as a function of single-scattering angle (SSA) at $14.5 \mathrm{~km}$ (green) and $20.5 \mathrm{~km}$ (red). This figure uses OMPS/LP observations taken on 19 June 2014, from the same orbit shown in Fig. 3. The dotted black line represents the value of the cloud detection threshold for the $\ln \mathrm{R}$ curves.

The OMPS/LP viewing geometry produces high singlescattering angle (SSA) values for Southern Hemisphere measurements (up to $160^{\circ}$ ) and low SSA values for Northern Hemisphere measurements (down to $20^{\circ}$ ). This relationship leads to large variations in ASI values over an orbit due to Mie scattering phase function effects. Figure 4 shows the variation of $\ln \mathrm{R}$ and ASI as a function of SSA at 14.5 and $20.5 \mathrm{~km}$ for the same orbit presented in Fig. 3. ASI values increase rapidly for $\mathrm{SSA}<80^{\circ}$ at both altitudes. In contrast, $\ln \mathrm{R}$ values are essentially constant throughout the orbit and are well below our cloud detection threshold except for the tropical region that is consistent with CALIPSO cloud detec- tions. We therefore use a constant cloud detection threshold to evaluate all LP measurements.

\subsection{Comparison with LP Version 2 results}

The cloud detection algorithm used in the OMPS/LP Version 2 ozone product (which is available at https://ozoneaq.gsfc. nasa.gov/data/omps) is based on the identification of sharp radiance profile changes at selected individual wavelengths (Rault and Loughman, 2013). Figure 5 shows cloud-top heights derived by our new radiance gradient algorithm and the LP Version 2 algorithm for a single orbit on 16 August 
(a) CALIPSO $532 \mathrm{~nm}$ backscatter

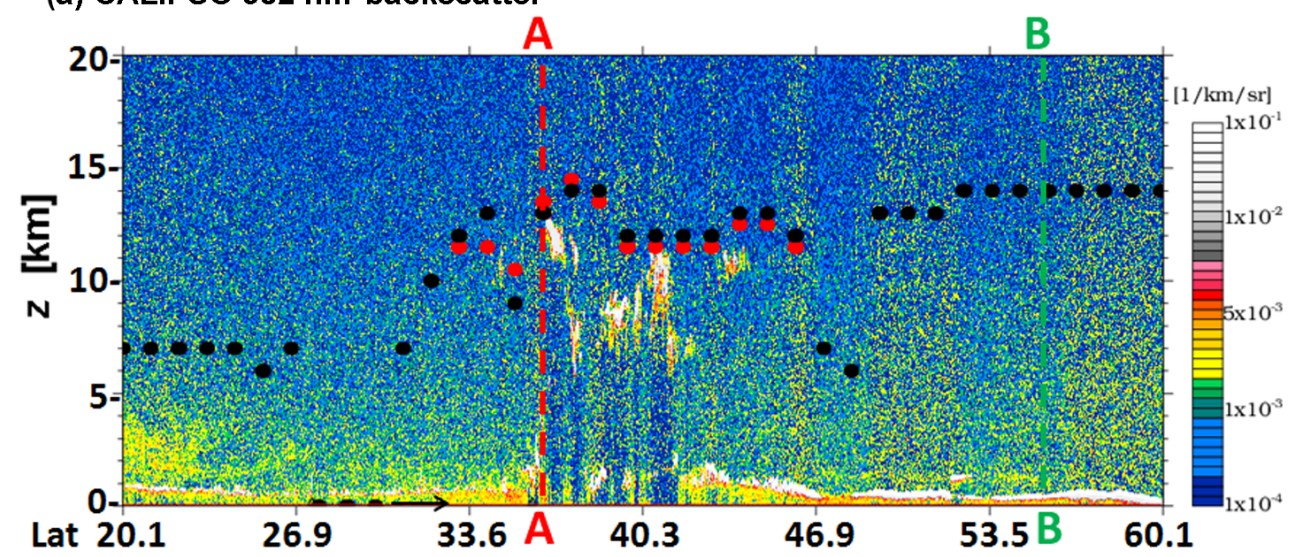

(b) Radiance profiles

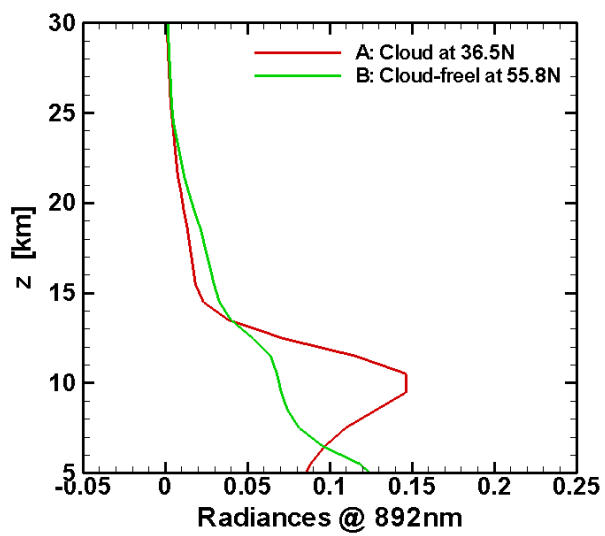

(c) Radiance gradient difference profiles

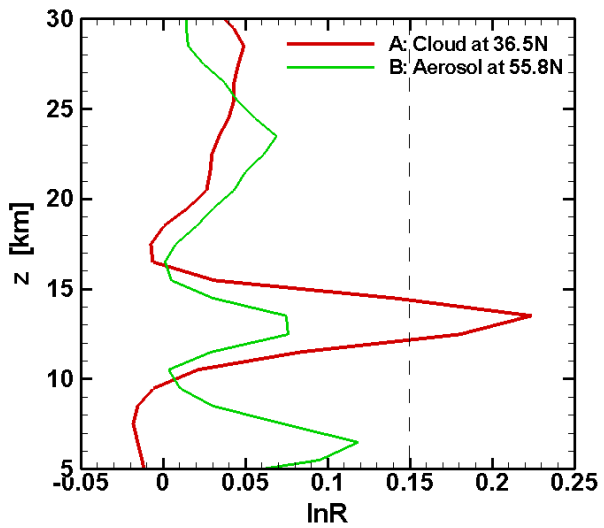

Figure 5. Comparison of LP cloud detection results for a single orbit on 16 August 2012. (a) CALIPSO $532 \mathrm{~nm}$ daytime backscatter data for the same orbit. The red-gray-white regions in the image denote the cloud layers. The red and black dots in the image represent cloud-top heights derived from the LP radiance gradient algorithm and the LP Version 2 algorithm, respectively. Lines A and B indicate OMPS/LP measurements at 36.5 and $55.8^{\circ} \mathrm{N}$, respectively. (b) Radiance profiles at $892 \mathrm{~nm}$ used as the basis for LP Version 2 algorithm cloud identification. Red: event A; green: event B. (c) Radiance gradient difference profiles used for new LP algorithm cloud identification. Red: event A; green: event B. The dashed line represents the value of the cloud detection threshold.

2012, with comparisons to the CALIPSO $532 \mathrm{~nm}$ backscatter coefficient for the same orbit. The LP Version 2 algorithm identifies many clouds that are not seen by CALIPSO, while the radiance gradient method only finds a few higher clouds between 33 and $46^{\circ} \mathrm{N}$ at locations where CALIPSO also shows such clouds. This suggests that the LP Version 2 algorithm may misidentify aerosols as clouds. To further illustrate this result, we focus on two selected events at $36.5^{\circ} \mathrm{N}$ (event A) and $55.8^{\circ} \mathrm{N}$ (event B). The LP Version 2 algorithm finds sufficiently sharp changes in $892 \mathrm{~nm}$ radiance profiles to identify clouds at $14.5 \mathrm{~km}$ for both events (Fig. $5 \mathrm{~b}$ ). In contrast, the radiance gradient algorithm finds a clear cloud signature in $\ln \mathrm{R}$ values for event $\mathrm{A}$, but a much weaker signature that falls below the detection threshold for event $\mathrm{B}$ (Fig. 5c). These results give us confidence that the radiance gradient algorithm is not creating "false-positive" cloud iden- tifications. Removing the incorrect cloud detections will also provide increased sampling in the upper troposphere for LP retrieval products.

\section{Validation of LP cloud height product}

In order to quantify the accuracy of LP cloud-top height values derived by the new LP radiance gradient algorithm, we evaluate our results against the CALIPSO VFM daytime product. The similarity in orbits between the SNPP and CALIPSO spacecraft makes it possible to select many events with reasonably tight coincidence criteria ( $\Delta$ latitude $< \pm 0.15^{\circ}, \Delta$ longitude $< \pm 3.25^{\circ}, \Delta$ time $<$ $\pm 1 \mathrm{~h}$ ). Only the CALIPSO measurements within the footprint of the S-NPP orbit have been considered. These re- 


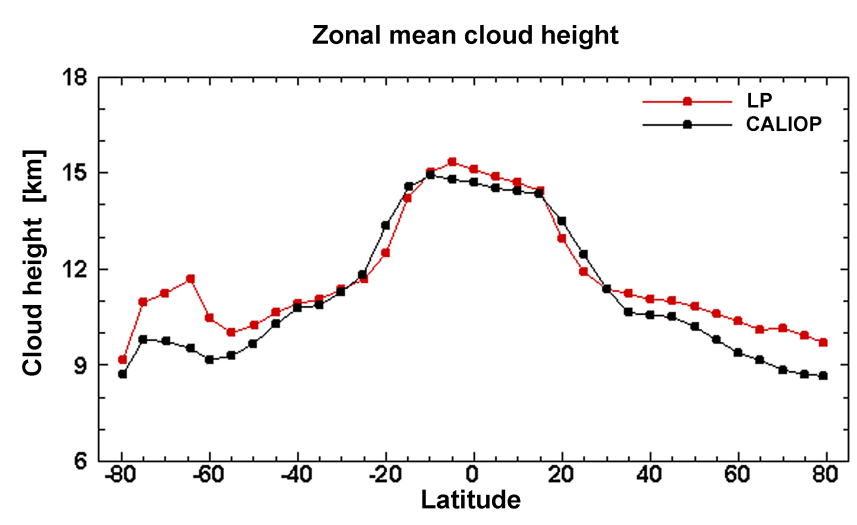

Figure 6. Zonal mean cloud height calculated from LP cloud detection algorithm results (red line) and collocated CALIPSO data (black line) in $5^{\circ}$ latitude bands. Results averaged over 70 sample days between April 2012 and February 2015.

quirements yielded approximately 439000 cases spread over 70 sample days between April 2012 and February 2015. We do not consider LP cloud detections below $5 \mathrm{~km}$ because our approach is not effective at such low altitudes.

Figure 6 shows the latitude distribution of cloud-top heights from these coincidence data sets in $5^{\circ}$ zonal mean latitude bands. The cloud-top heights derived from the LP algorithm agree quite well with CALIPSO data in the tropics and midlatitudes (up to approximately $50^{\circ}$ ). The cloud altitudes derived from both data sets decrease towards the poles due to the general decrease of the tropopause height. The LP cloud height values are higher in polar regions because our data set consistently includes PSCs, which are identified at $15-30 \mathrm{~km}$ in winter and spring months (see example in Fig. 2). LP measurements may also detect clouds that are located at different positions along the line of sight, which would give lower derived cloud heights than if the same cloud were located at the tangent point position.

Figure 7 shows a histogram of cloud height differences between the LP and CALIPSO data sets. The difference values are calculated as the LP cloud-top height minus the collocated CALIPSO value. The histogram has been constructed using bins of $1 \mathrm{~km}$, the vertical sampling of the LP measurements. The most common difference values occur between -1 and $+4 \mathrm{~km}$, with a median difference of $\Delta z_{\text {cloud }}=$ $1.8 \mathrm{~km}$. A Gaussian fit to these data yields a similar median difference value $(2.0 \mathrm{~km})$. We note that the LP cloud detection algorithm identifies the upper edge of a cloud, so it is not surprising to find a high bias in reported heights relative to CALIPSO cloud height values based on nadir-viewing lidar measurements. In addition, the LP vertical resolution is $\sim 1.6-1.8 \mathrm{~km}$, whereas CALIPSO data have much finer vertical sampling and resolution. The extended tail of this distribution towards large negative values corresponds to scattered high-cloud values $\left(z_{\text {cloud }}>20 \mathrm{~km}\right)$ in the CALIPSO data set.

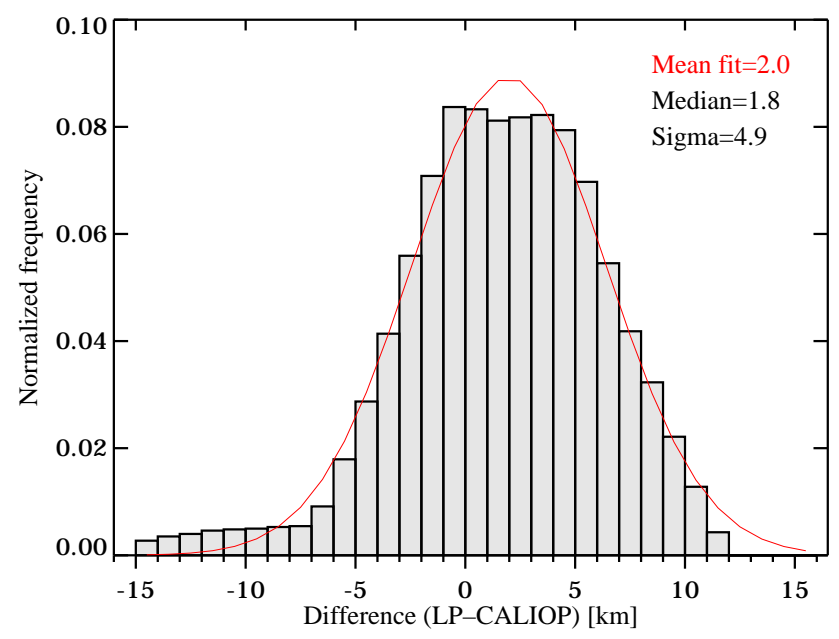

Figure 7. Normalized frequency histogram of all cloud height differences (LP-CALIPSO) from coincidence data sets in $\Delta z_{\text {cloud }}=$ $1 \mathrm{~km}$ intervals. The red curve represents a Gaussian fit to the data.

Figure 8 shows two histograms of cloud-top heights in the tropics as detected from the LP algorithm and from CALIPSO data. These distributions have very similar shapes, and the distributions are roughly Gaussian. The maximum cloud height occurrence frequency is observed between 14 and $16 \mathrm{~km}$ for both instruments. We note that the CALIPSO data show some clouds up to $25 \mathrm{~km}$ height, which confirms previous studies that CALIPSO can sometimes misidentify aerosols as clouds (Chen et al., 2010, 2012). However, the LP data set does identify a population of clouds at $20-22 \mathrm{~km}$, which are clearly above the tropopause when individual orbits are inspected. The presence of these unusually high clouds in the tropics is connected with Kelut Volcano, which erupted in February 2014. Remember that the $\ln \mathrm{R}$ value calculation presented in Sect. 2 determines the slope of the radiance gradient. Larger aerosol particles, such as those found in fresh volcanic plumes, will increase the slope of the radiance gradient and makes these events more difficult to distinguish from "normal" clouds. In addition, patchy clouds in the near and far sides of the tangent point may also cause biased estimates of cloud height. This potential error source was investigated in detail by Kent et al. (1997).

\section{Summary and conclusions}

We have developed a revised cloud detection algorithm for use with OMPS/LP measurements. This algorithm uses the spectral dependence of the vertical gradient of radiance at 674 and $868 \mathrm{~nm}$ to identify clouds and distinguish them from aerosols. Comparison of cloud detection results for individual events with CALIPSO data confirms the success of this approach. The revised LP cloud detection algorithm is also more effective than the LP Version 2 algorithm in identifying only valid clouds. Our cloud detection results are consis- 

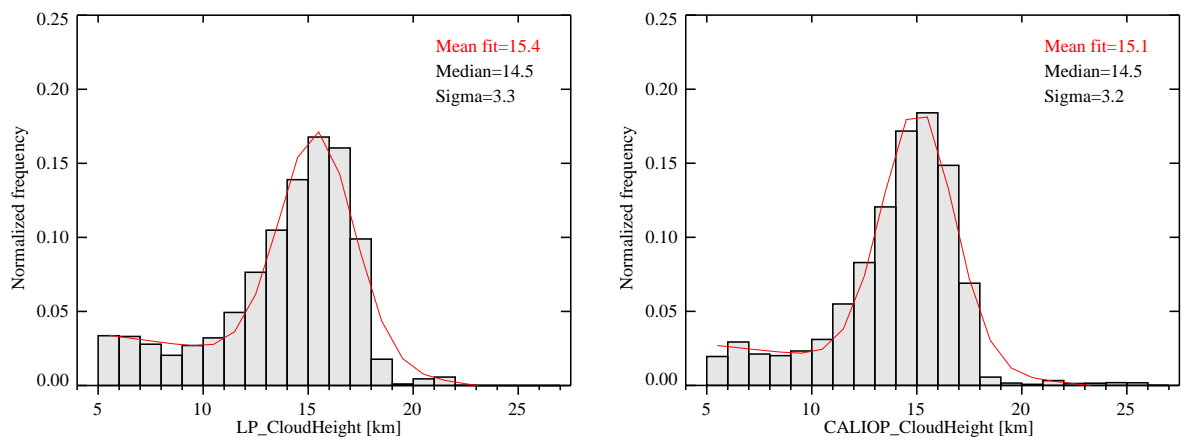

Figure 8. Normalized frequency histograms of all cloud height values from LP cloud detection results (left panel) and the collocated CALIPSO data (right panel) in the tropics (latitude $< \pm 30^{\circ}$ ) in $\Delta z_{\text {cloud }}=1 \mathrm{~km}$ intervals. The red curves represent a linear combination of a Gaussian and quadratic function fit to each data set.

tent with CALIPSO observations in terms of latitude dependence and frequency distribution of altitudes. The offset in absolute cloud height for coincident measurements is consistent with differences between the detection methods. The LP cloud detection algorithm also consistently identifies polar stratospheric clouds in both hemispheres, which may be useful for directly examining the impact of PSCs on LP ozone retrievals. We do not attempt to retrieve cloud heights below $5 \mathrm{~km}$ with this algorithm. Aerosol layers with larger particles, such as fresh volcanic plumes, are more likely to be classified as clouds. Further theoretical studies of spectral properties and scattering effects are needed to fully understand the applicability range and limitations of this method. The new cloud detection algorithm will be implemented for the forthcoming LP Version 3 ozone and aerosol retrieval algorithms, and the LP cloud height values will also be distributed as a public data product.

\section{Data availability}

The OMPS/LP Level 1 gridded radiance product (LP-L1GEV) used to create the cloud height product described in this paper can be obtained at https://ozoneaq.gsfc.nasa.gov/data/ ozone/, 2016.

Acknowledgements. We thank Mark Schoeberl for his insightful comments on the development of this algorithm. Zhong Chen and Matthew DeLand were supported by NASA contract NNG12HP08C.

Edited by: D. Loyola

\section{References}

Bourassa A. E., Degenstein, D. A., and Llewellyn E. J.: Climatology of the subvisual cirrus clouds as seen by OSIRIS on Odin, Adv. Space Res., 36, 807-812, 2005.
Chen, B., Huang, J., Minnis, P., Hu, Y., Yi, Y., Liu, Z., Zhang, D., and Wang, X.: Detection of dust aerosol by combining CALIPSO active lidar and passive IIR measurements, Atmos. Chem. Phys., 10, 4241-4251, doi:10.5194/acp-10-4241-2010, 2010.

Chen, Z., Torres, O., McCormick, M. P., Smith, W., and Ahn, C.: Comparative study of aerosol and cloud detected by CALIPSO and OMI, Atmos. Environ., 51, 187-195, 2012.

Eichmann, K.-U., Lelli, L., von Savigny, C., Sembhi, H., and Burrows, J. P.: Global cloud top height retrieval using SCIAMACHY limb spectra: model studies and first results, Atmos. Meas. Tech., 9, 793-815, doi:10.5194/amt-9-793-2016, 2016.

Flynn, L. E., Seftor, C. J., Larsen, J. C., and Xu, P.: The Ozone Mapping and Profiler Suite, in: Earth Science Satellite Remote Sensing, edited by: Qu, J. J., Gao, W., Kafatos, M., Murphy, R. E., and Salomonson, V. V., Springer, Berlin, 279-296, doi:10.1007/9783-540-37293-6, 2007

Herman, B. M., Caudill, T. R., Flittner, D. E., Thome, K. J., and Ben-David, A.: Comparison of the Gauss-Seidel spherical polarized radiative transfer code with other radiative transfer codes, Appl. Optics, 34, 4563-4572, 1995.

Jaross, G., Bhartia, P. K., Chen, G., Kowitt, M., Haken, M., Chen, Z., Xu, P., Warner, J., and Kelly, T.: OMPS Limb Profiler instrument performance assessment, J. Geophys. Res.-Atmos., 119, 4399-4412, doi:10.1002/2013JD020482, 2014.

Kacenelenbogen, M., Vaughan, M. A., Redemann, J., Hoff, R. M., Rogers, R. R., Ferrare, R. A., Russell, P. B., Hostetler, C. A., Hair, J. W., and Holben, B. N.: An accuracy assessment of the CALIOP/CALIPSO version 2/version 3 daytime aerosol extinction product based on a detailed multi-sensor, multiplatform case study, Atmos. Chem. Phys., 11, 3981-4000, doi:10.5194/acp-11-3981-2011, 2011.

Kent, G. S., Winker, D. M., Vaughan, M. A., Wang, P.H., and Skeens, K. M.: Simulation of Stratospheric Aerosol and Gas Experiment (SAGE) II cloud measurements using airborne lidar data, J. Geophys. Res., 102, 21795-21807, doi:10.1029/97JD01390, 1997.

Koelemeijer, R. B. A., Stammes, P., Hovenier, J. W., and de Haan, J. F.: A fast method for retrieval of cloud parameters using oxygen A-band measurements from the Global Ozone Monitoring Experiment, J. Geophys. Res., 106, 3475-3490, doi:10.1029/2000JD900657, 2001. 
Kokhanovsky, A. A., Rozanov, V. V., Burrows, J. P., Eichmann, K. U., Lotz, W., and Vountas, M.: The SCIAMACHY cloud products: Algorithms and examples from ENVISAT, Adv. Space Res., 36, 789-799, doi:10.1016/j.asr.2005.03.026, 2005.

Kuze, A. and Chance, K. V.: Analysis of cloud top height and cloud coverage from satellites using the $\mathrm{O}_{2} \mathrm{~A}$ and $\mathrm{B}$ bands, J. Geophys. Res., 99, 14481-14491, 1994.

Loyola, D., Thomas, W., Livschitz, Y., Ruppert, T., Albert, P., and Hollmann, R.: Cloud properties derived from GOME/ERS-2 backscatter data for trace gas retrieval, IEEE T. Geosci. Remote, 45, 2747-2758, 2007.

Loyola, D., Thomas, W., Spurr, R., and Mayer, B.: Global patterns in daytime cloud properties derived from GOME backscatter UV-VIS measurements, Int. J. Remote Sens., 31, 4295-4318, 2010.

OMPS/LP Level 1: OMPS Limb Profiler Suomi NPP-LP-L1G-EV, available at: https://ozoneaq.gsfc.nasa.gov/data/ozone/, last access: 22 March 2016.

Rault, D. F. and Loughman, R. P.: The OMPS Limb Profiler Environmental Data Record algorithm theoretical basis document and expected performance, IEEE T. Geosci. Remote, 51, 2505-2527, 2013.

Rozanov, V. V. and Kokhanovsky, A. A.: Semianalytical cloud retrieval algorithm as applied to the cloud top altitude and the cloud geometrical thickness determination from top-of-atmosphere reflectance measurements in the oxygen A band, J. Geophys. Res., 109, D05202, doi:10.1029/2003JD004104, 2004.
Schuessler, O., Loyola, D., Doicu, A., and Spurr, R.: Information Content in the Oxygen A-Band for the Retrieval of Macrophysical Cloud Parameters, IEEE T. Geosci. Remote, 52, 3246-3255, 2014.

van Diedenhoven, B., Hasekamp, O. P., and Landgraf, J.: Retrieval of cloud parameters from satellite-based reflectance measurements in the ultraviolet and the oxygen A-band, J. Geophys. Res., 112, D15208, doi:10.1029/2006JD008155, 2007.

Vaughan, M., Young, S., Winker, D., Powell, K., Omar, A., Liu, Z., $\mathrm{Hu}, \mathrm{Y}$., and Hostetler, C.: Fully automated analysis of spacebased lidar data: an overview of the CALIPSO retrieval algorithms and data products, Proc. SPIE, 5575, 16-30, 2004.

von Savigny, C., Ulasi, E. P., Eichmann, K.-U., Bovensmann, H., and Burrows, J. P.: Detection and mapping of polar stratospheric clouds using limb scattering observations, Atmos. Chem. Phys., 5, 3071-3079, doi:10.5194/acp-5-3071-2005, 2005.

Winker, D. M., Pelon, J., and McCormick, M. P.: The CALIPSO mission: Spaceborne lidar for observation of aerosols and clouds, Proc. SPIE, 4893, 1-11, 2003. 